\title{
Impact of Vehicular Array Position on Urban MIMO Channel Characteristics
}

\author{
Christopher C. Squires and Tricia J. Willink \\ Communications Research Centre, Ottawa, ON, Canada K2H $8 \mathrm{~S} 2$ \\ Correspondence should be addressed to Christopher C. Squires, chris.squires@crc.gc.ca
}

Received 24 December 2010; Revised 10 March 2011; Accepted 18 July 2011

Academic Editor: Z. Chen

Copyright ( $) 2011$ C. C. Squires and T. J. Willink. This is an open access article distributed under the Creative Commons Attribution License, which permits unrestricted use, distribution, and reproduction in any medium, provided the original work is properly cited.

\begin{abstract}
MIMO channel measurements have been performed in urban environments with antenna arrays positioned on the roof and sides of the measurement vehicle. Analysis of the data shows that the array's position on the vehicle, along with the location and orientation of the vehicle with respect to the transmitter, can have a considerable impact on the channel characteristics observed. Array elements on the vehicle's roof receive the same multipath components, and, therefore, have identically distributed channel responses. However, the characteristics of the multipath components observed at each side of the vehicle can differ significantly. Specifically, shadowing from the vehicle combined with the directionality of the side-mounted array elements affects the angular distribution and powers of the received multipath components, affecting the achievable diversity and channel capacity. This can have significant impact on system performance and should be considered when designing and analyzing vehicular-based signalling techniques.
\end{abstract}

\section{Introduction}

The capability of multiple-input multiple-output (MIMO) communications systems to exploit multipath diversity to improve system throughput and robustness has been extensively reported on since the early work in $[1,2]$. A detailed review can be found in [3]. The signalling strategy used to achieve the desired performance depends on the characteristics of the channel. In particular, the spatial corre-la-tion observed depends on the number and angular diversity of the multipath components arriving at the receiver [4]; this changes as mobile terminals move through the physical environment. Thus, to determine the expected performance of a MIMO system, it is necessary to have a good representation of the channel characteristics.

Many models of MIMO systems have been developed based on assumptions about the physical structure of the scattering environment, for example, ring models [5] and geometrically based stochastic models [6]. For a summary of these types of models, see [7, Chapter 4]. These models typically assume that the antenna elements at the receiving array have an unobstructed view of the scatterers. For vehicular applications, the antenna elements are close enough to observe the same set of scatterers; hence, the fading statistics and shadowing are the same at all antenna elements. When the array elements are separated by larger spacings, for example, in distributed array systems, the system becomes more robust to variations in shadowing and system capacity can be improved [8].

Although the importance of a well-designed channel model cannot be understated in the analysis of signalling strategies, data gathered through measurements and experimentation often reveal propagation conditions that are significantly more challenging to operate in than those resulting from simulation. Being aware of these real world signalling conditions can assist in understanding unexpected system behaviour or performance.

Much insight has been gained into the characteristics of real MIMO channels through extensive measurement campaigns. Studies of vehicular-based MIMO channels were reported in [9-17], where the MIMO channels were measured using systems with one fixed endpoint and one vehicular-based endpoint. Lower mobility outdoor measurement campaigns have been performed using a mobile transmitter or receiver mounted on a trolley, pushed at walking speed, for example, [18-20]. The fixed wireless outdoor 
MIMO channel has also been investigated in urban environments, see, for example, [21-25]. Further, experiments in which both ends of the wireless link are mobile have been performed to measure vehicle-to-vehicle MIMO channels, as reported in [26-30].

A thorough survey of the literature covering vehicularbased MIMO measurement campaigns reveals a common configuration at the mobile: the antennas are mounted on the top of the vehicle or trolley, maximizing the area over which each array element receives signal energy and minimizing the effect of the vehicle on the channel measurements. However, as vehicular-based multielement communication systems become more common, communications engineers are faced with practical and aesthetic restrictions in which antennas must be mounted in positions considered less than optimal from a propagation perspective. Specifically, roofmounted antennas are not practical in many applications; rather, antenna elements may be integrated into the body panels to maintain the vehicle's aerodynamic style. In this case, the antenna elements do not have an unobstructed view of the scattering and reflecting environment, and, as a result, differences in the simultaneous fading characteristics may be observed at different positions of the vehicle.

Another practical restriction to vehicular-based MIMO systems stems from the size requirement for multielement array antennas. Colocated orthogonally polarized array elements have been considered as a means to reduce array dimensions without increasing correlation between elements $[11,14,18,20,21,31]$. The propagation characteristics of orthogonally polarized electromagnetic waves result in exploitable diversity, even when the elements are located close together [32, Chapter 13]. However, it has been observed that there is a loss of power on the cross-polarized channels, resulting in a reduction in the achievable diversity and limiting the overall performance gain [31].

In this investigation, we compare MIMO channels measured with a roof-mounted array to channels measured with the array elements mounted to the sides of a vehicle. Consistent with the usual measurement approach, the first set of measurements was obtained using a linear array of omnidirectional quarter-wavelength monopoles positioned on the top of the vehicle. In this case, each antenna is exposed to signal energy arriving from $360^{\circ}$ in azimuth and shadowing from the vehicle is minimized. To characterize the MIMO channel observed when the vehicle roof top is not available for antenna installations, a second set of measurements was obtained using linear arrays of dualpolarized antennas attached to the driver and passenger sides of the vehicle. However, when the array elements are positioned in this manner, the reduced angular range over which each array element receives signal energy, combined with increased obstruction or shadowing by the vehicle, results in a greater attenuation of the received signal and fewer exploitable multipath components.

For the work reported herein, channel measurements were obtained using the Communications Research Centre (CRC) MIMO measurement system in urban Ottawa, Canada (Section 2). Analysis of these data reveals that the signal components received are strongly dependent on the position and polarization of the array (Section 3). To evaluate the impact of the array position on MIMO system performance, the characteristics of the received power (Section 4) and spatial diversity (Section 5) are investigated. These two factors determine the channel capacity (Section 6), which is often used as a measure of idealized MIMO system performance. As a result of this analysis, we conclude in Section 7 that, when the antenna array cannot be placed on the roof, the array elements should be placed around the vehicle to minimize the impact of vehicular shadowing. However, with such an antenna arrangement, the total power and the number of multipath components received at each array element may differ significantly. For these scenarios, modified MIMO models are required that incorporate time varying differences in the propagation characteristics at each array position.

\section{Measurement System}

Measurements of urban MIMO channels were performed in downtown Ottawa, Canada, in April, 2008, using the CRC MIMO channel sounder described in detail in [12]. The trailer-mounted transmitter was parked along Lyon Street, as shown in Figure 1, with the antenna array mounted on a mast about $3 \mathrm{~m}$ above street level and oriented such that the array axis was perpendicular to the street. The linear array was comprised of $N_{t}=4$ quarter-wavelength drooping radial monopole antennas spaced by one wavelength, $\lambda$. A repeating pseudonoise sequence was transmitted simultaneously from each array element at a chip rate of $12.5 \mathrm{Mcps}$ and a centre frequency of approximately $2 \mathrm{GHz}$.

At the receiver, two sets of measurements were performed using arrays mounted on the top and sides of the measurement vehicle. For the first measurements, a linear array of $N_{r}=4$ quarter-wavelength drooping radial monopole antennas was mounted on top of the vehicle, at a height of approximately $2 \mathrm{~m}$ above street level and with the axis of the array positioned perpendicular to the direction of travel (see Figure 2). A photograph showing how the antennas were positioned is shown in Figure 3. The antenna elements in this array have a theoretical gain of approximately $5 \mathrm{dBi}$ and a pattern that is omnidirectional in azimuth but not in elevation. Roof-mounted antennas of this type have been used extensively in characterizing mobile radio channels, for example, $[33,34]$, and have been shown $[33,35]$ to have an azimuthal antenna pattern that is within $\pm 3 \mathrm{~dB}$ of omnidirectional.

The second set of measurements was made using two $N_{r}=4$-element linear arrays with $1 \lambda$ spacing. One array was mounted on the driver side of the vehicle and the other on the passenger side. Each array was positioned approximately $1 \mathrm{~m}$ from the street level. Both the driver side array (DSA) and passenger side array (PSA) consisted of dual-polarized (vertically and horizontally) patch antennas with thick ground planes to provide robustness, a gain of $8.5 \mathrm{dBi}$ and a $3 \mathrm{~dB}(10 \mathrm{~dB})$ beam width of $72^{\circ}\left(134^{\circ}\right)$ in both azimuth and elevation. An RF switch, mounted under each patch element, was used to alternate measurements from each polarization; for more detail, see [36]. A depiction of the 


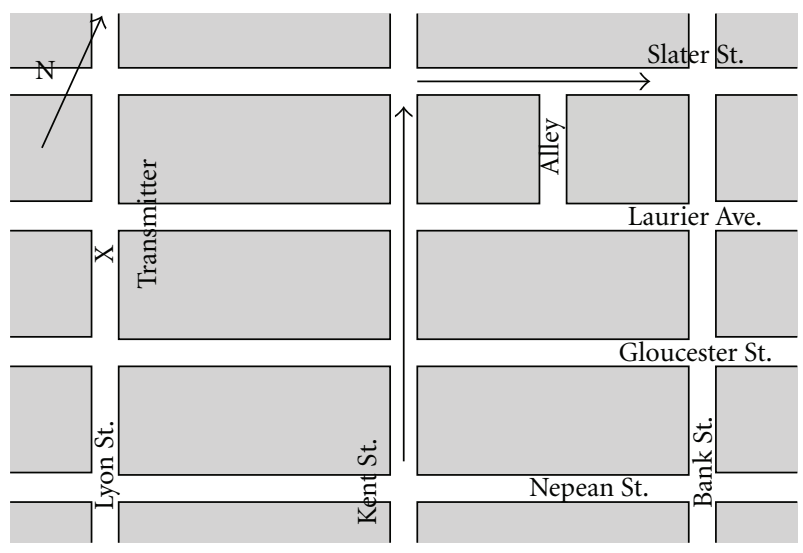

Figure 1: Map of the measurement areas in downtown Ottawa. The arrows indicate the measurement routes, and $\mathrm{x}$ marks the transmitter location.

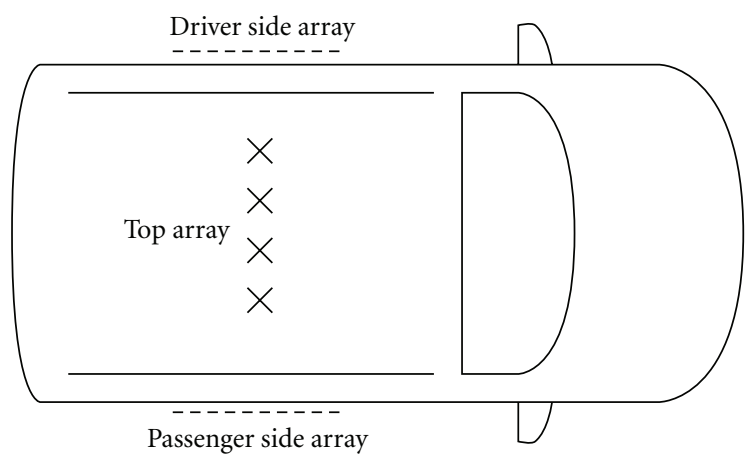

FIgURE 2: Positions of the top, driver side, and passenger side arrays on the measurement vehicle.

arrangement of the arrays on the vehicle is shown in Figure 2; see also the photograph in Figure 3.

The MIMO receiver consists of separate RF chains operating synchronously to downconvert the received signals to baseband. At the top array (TA) and side arrays (SA), short snapshots of the received baseband signals were sampled at 50 Msps. A new snapshot was acquired every $T_{s}=2 \mathrm{~ms}$ at the TA and every $2 \mathrm{~ms}$ at each SA, alternating between polarizations, providing a snapshot rate of $T_{s}=4 \mathrm{~ms}$ for each polarization. For a mobile receiver travelling at a speed of $8.3 \mathrm{~m} / \mathrm{s}$, this snapshot rate is approximately 8 times and 4 times the maximum Doppler rate for the TA and SA, respectively, exceeding the Nyquist rate. The measurements on the vertical polarizations (VP) and horizontal polarizations (HP) can therefore be considered almost simultaneous for the channel characteristics considered. The raw sampled data from each acquisition were stored on PC hard drives and postprocessed by correlating with the transmitted sequence to generate a channel impulse response estimate for each of the $N_{t} N_{r}$ links. Narrowband channel response estimates were then generated by extracting the complex value corresponding to a given frequency point from the FFTs of the impulse response estimates; these

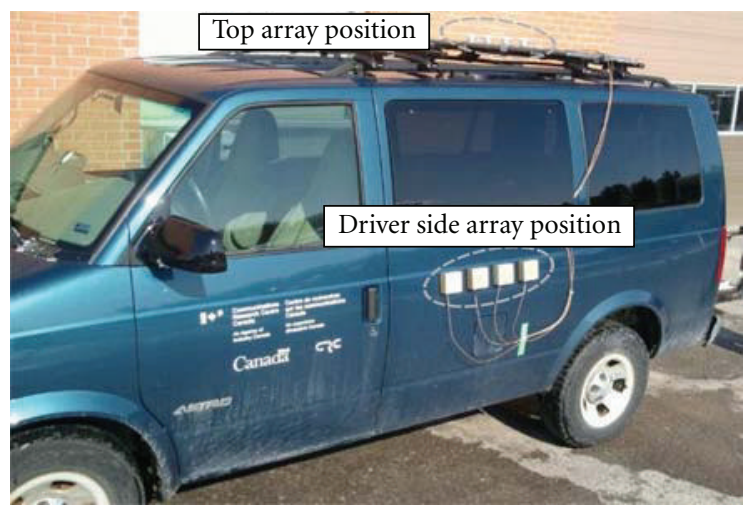

FIgure 3: Photograph of the measurement vehicle showing the array positions on the top and side with the protective radomes removed.

narrowband channel responses are assumed to be frequency flat.

The channel measurements were acquired within two blocks or $0.5 \mathrm{~km}$ of the transmitter by driving the receiver along specific measurement routes at a nearly constant speed of $8.3 \mathrm{~m} / \mathrm{s}$. These routes are considered to be high-density urban environments with office and apartment buildings of heights considerably greater than the transmit or receive antennas. The top- and side-mounted antenna arrays could not be used simultaneously; hence, the routes were driven multiple times using either the TA or both the DSA and PSA. All measurements were performed between mid-morning and mid-afternoon when traffic was low to moderate. Two of the measurement routes, indicated in Figure 1, are discussed herein as they are typical of routes running both north-south and east-west.

2.1. Channel Representation. Typically, MIMO channel models are developed based on the assumption that the antenna elements are separated enough to reduce spatial correlation but colocated such that the complex fading coefficients for each transmit-receive element pair share a common statistical distribution. For very large separations between array elements or when directional or polarized elements are used, assumptions of homogeneous fading characteristics may not hold [8]. Similar heterogeneous fading characteristics may occur in vehicular-based MIMO systems operating in urban environments where the positions chosen to mount the array elements, along with the relative location of the mobile to the transmitter, will impact the number of multipath components received at each element, as well as their corresponding powers and directions of arrival [37].

The MIMO channel response matrix $\mathbf{H}$, sampled at time intervals $t=n T_{s}$, is represented by an $N_{r} \times N_{t}$ matrix of complex channel coefficients

$$
\mathbf{H}^{(i, j)}[n]=\left[h_{r, t}^{(i, j)}[n]\right]_{N_{r} \times N_{t}},
$$

such that $r=1,2, \ldots, N_{r}$ and $t=1, \ldots, N_{t}$, and $h_{r, t}^{(i, j)}$ is the complex fading coefficient between transmit element $t$ and 
receive element $r$. The polarization of the receive antenna is indicated by $i=\{v, h\}$, where $\{v, h\} \triangleq\{$ vertical, horizontal $\}$. The position of the array is defined by $j=\{T, D, P\}$ as the top $(T)$, driver side $(D)$, or passenger side $(P)$.

To preserve the power difference between the TA and the SAs, the data collected at each array position were normalized with respect to the data collected at the TA using the normalization factor

$$
\eta=\sqrt{\frac{1}{N_{r} N_{t} N} \sum_{n=1}^{N}\left\|\mathbf{H}^{(v, T)}[n]\right\|_{F}^{2}},
$$

where $N$ is the total number of samples in the measured time series and $\|\cdot\|_{F}$ is the Frobenius norm. The focus of this investigation is to provide insight into the differences in the effective channels observed by a real communication system at each array position. As such, no effort has been made to remove the effects of the antenna radiation patterns or crosscoupling between array elements.

To estimate characteristics of the observed radio channel, the underlying statistical processes must be at least widesense stationary. However, for mobile systems these statistical processes vary with time as the multipath structure (number, power, and angles of arrival) observed at the receiver changes. The estimation of channel metrics must therefore be restricted to intervals over which a reasonable assumption of wide-sense stationarity can be made. Techniques for assessing the stationarity of observed channel measurements have been discussed in [38, 39]. In [40], MUSIC analysis was used to detect changes in the number of multipath wave fronts impinging on a synthetic array along with their associated powers and angles of arrival. A statistical test was introduced in [41] based on the Doppler periodogram. Both $[40,41]$, which used measurement data collected at similar frequencies and in a similar environment to that considered herein, indicated that intervals of $2 \mathrm{~m}$ could generally be treated as wide-sense stationary. Using these results as a guideline and with the assumption that the vehicle speed remained constant over intervals of this length, the data were analyzed using sample segments equivalent to $2 \mathrm{~m}$ or approximately $13 \lambda$. For the TA, this is $N=120$ samples, and $N=60$ for the SAs.

\section{Doppler Spectrum}

The spatial characteristics of received signal energy in urban environments can change considerably over short distances. As the angles of arrival of individual multipath components change, some components are lost and others arise. Passing local physical features such as other vehicles may result in small scale changes, while others, for example, intersections or buildings, cause larger scale changes. In this section, the evolution of the Doppler spectrum is used to investigate the impact of array positions on the changes in multipath structure.

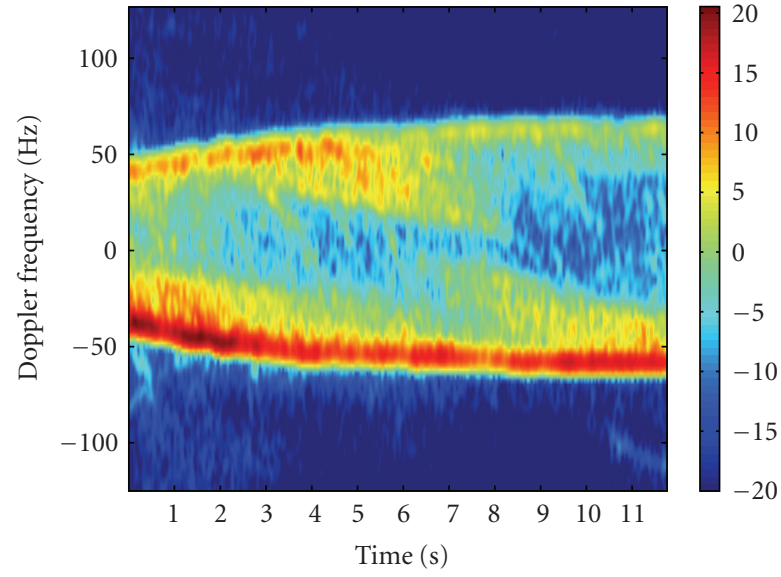

Figure 4: Doppler power (dB) spectrum measured at the top array on Slater Street.

The Doppler spectrum is estimated by the modified periodogram [42, Chapter 14],

$$
S_{r, t}^{(i, j)}(f)=\frac{1}{N U}\left|\sum_{n=0}^{N-1} h_{r, t}^{(i, j)}[n] w[n] e^{-j 2 \pi f n}\right|^{2},
$$

where $f=n / N T_{s}$ and $U$ is the normalization factor applied to the Blackman window,

$$
w[n]=0.42-0.5 \cos \left(\frac{2 \pi n}{N}\right)+0.08 \cos \left(\frac{4 \pi n}{N}\right) .
$$

The Blackman window improves the variance of the estimator by reducing spectral leakage resulting from using a finite length data sample. $S_{r, t}^{(i, j)}(f)$ is estimated using a sliding window of complex channel coefficients corresponding to $13 \lambda$ and averaged over all transmit-receive element pairs at each array position such that

$$
\bar{S}^{(i, j)}(f)=\frac{1}{N_{t} N_{r}} \sum_{t=1}^{N_{t}} \sum_{r=1}^{N_{r}} S_{r, t}^{(i, j)}(f) .
$$

Figures 4-7 show the time series of averaged Doppler spectra obtained for different arrays on two routes. The plots show the evolution of the received angular power as the vehicle progresses along the measurement routes. The power at frequency $f_{d}$ arrives at angle $\theta$, where $f_{d}=v / \lambda \cos \theta$, relative to the direction of travel of the mobile receiver. If a multipath component arrives from the static transmitter after interaction with static objects, its maximum frequency is $f_{m}= \pm 56.6 \mathrm{~Hz}$ at $v=8.3 \mathrm{~m} / \mathrm{s}$. This occurs when the energy arrives from directly in front of $\left(\theta=0^{\circ}\right)$ or behind $\left(\theta=180^{\circ}\right)$ the vehicle. A small number of the Doppler spectra exhibits components with frequency larger than $f_{m}$ : this is attributed to multipath components interacting with moving vehicles, increasing the effective relative velocity $v$. However, it is clear that most of the signal energy is received after interaction with static objects in the environment, such as buildings.

Figure 4 shows the time series of averaged Doppler spectra obtained at the TA along Slater Street where the 


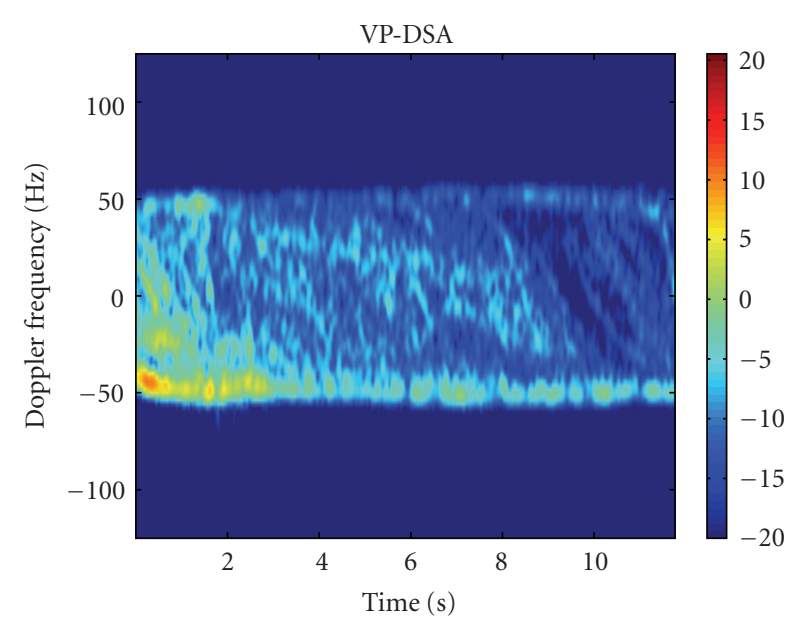

(a)

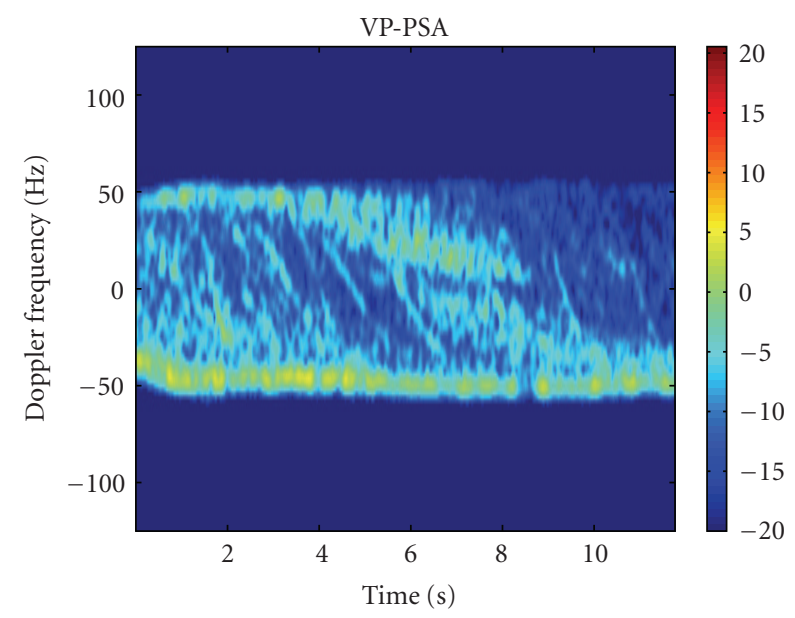

(c)

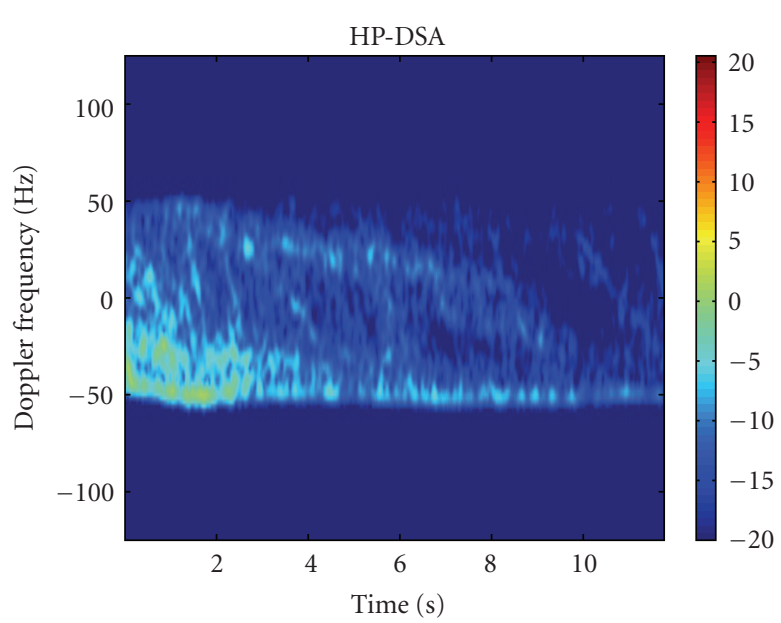

(b)

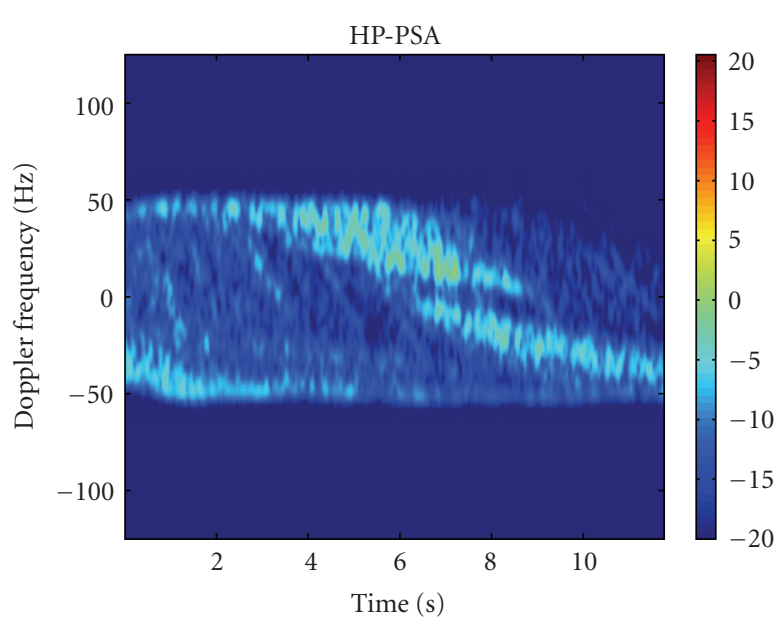

(d)

Figure 5: Doppler power (dB) spectra measured at the VP-DSA, HP-DSA, VP-PSA, and HP-PSA on Slater Street.

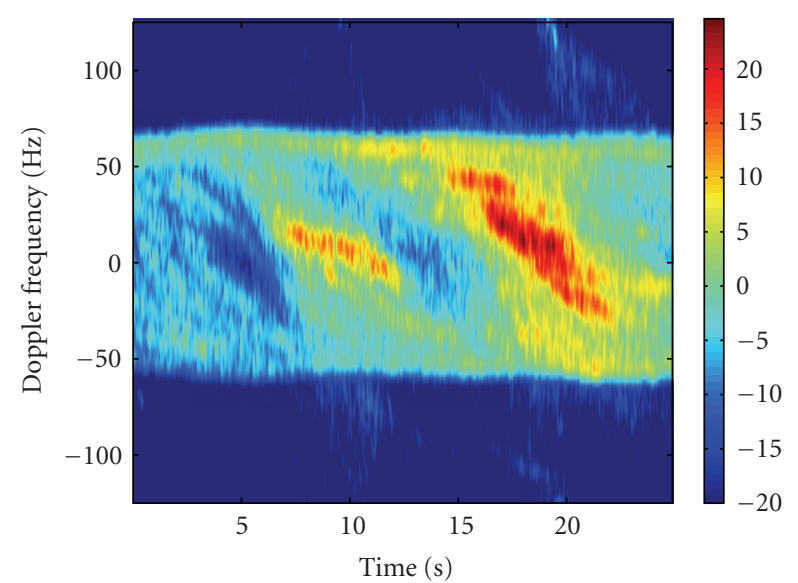

FIGURE 6: Doppler power (dB) spectrum measured at the top array on Kent Street.

vehicle is moving away from the transmitter through a narrow urban canyon. The signal arriving at the TA is dominated by a strong component arriving from the rear of the vehicle and, for the first $3 \mathrm{~s}$ of the measurement, a secondary, weaker source arriving from the front. After the $3 \mathrm{~s}$ point in the measurement, the angle of the forward component begins to transition to the side of the vehicle, and, by $8 \mathrm{~s}$ it has moved completely to the rear. This is indicative of the vehicle driving past a signal source, in this case, a service alley that connects Laurier Avenue to Slater Street between the office buildings. Signal energy travels through this alley by reflecting from the ground and the buildings on either side. The angles of entry of the signal energy into Slater Street are such that reduced signal energy is received from broadside $\left(\theta=90^{\circ}\right.$ and $\left.f_{d}=0\right)$ as the vehicle passes the entrance to the alley, at approximately 7-8 s.

The Doppler spectra obtained at each of the SAs are shown in Figure 5. These plots show how the combination of multipath components that form the TA spectrum are disjoint at the SAs. For example, the strong component arriving from near the rear is most prominent on the vertical polarization (VP) of both the DSA and PSA indicating that this component is highly polarized, as expected, as the main mechanisms for signal energy entering Slater Street from Lyon Street and Kent Street are diffraction around and 


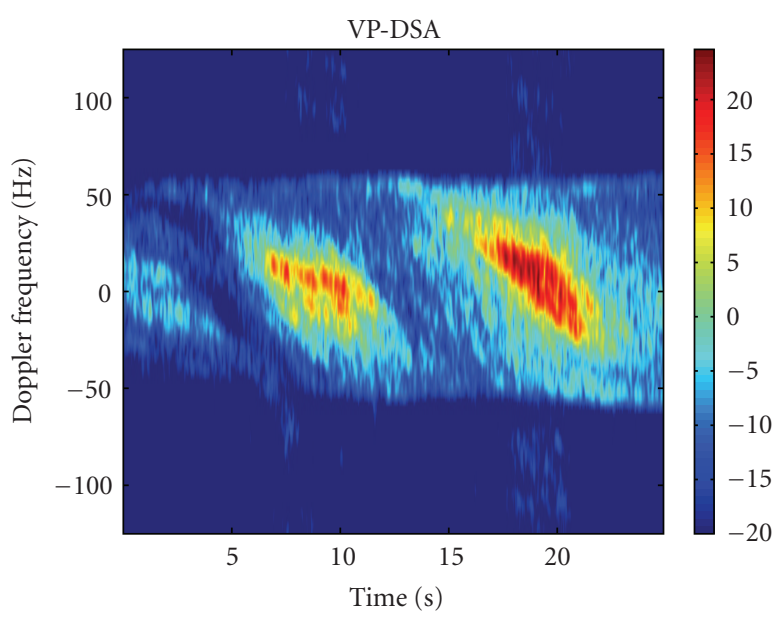

(a)

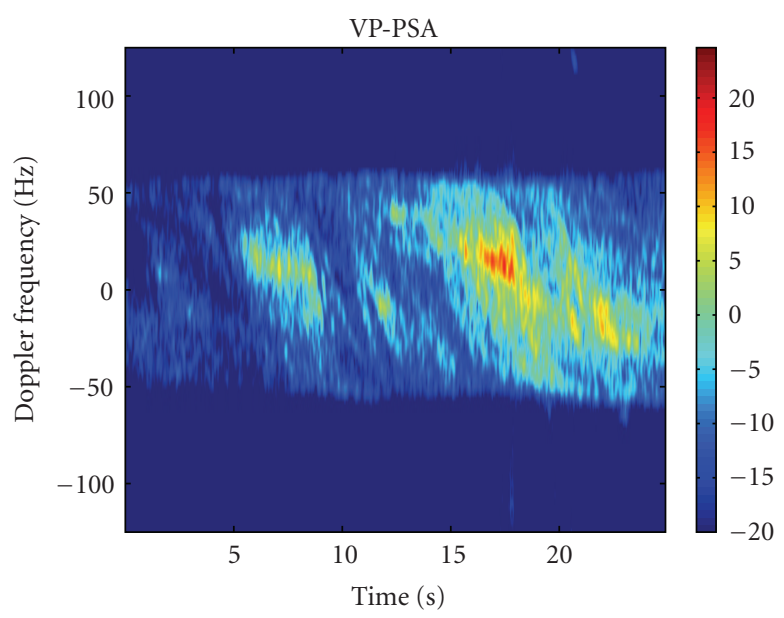

(c)

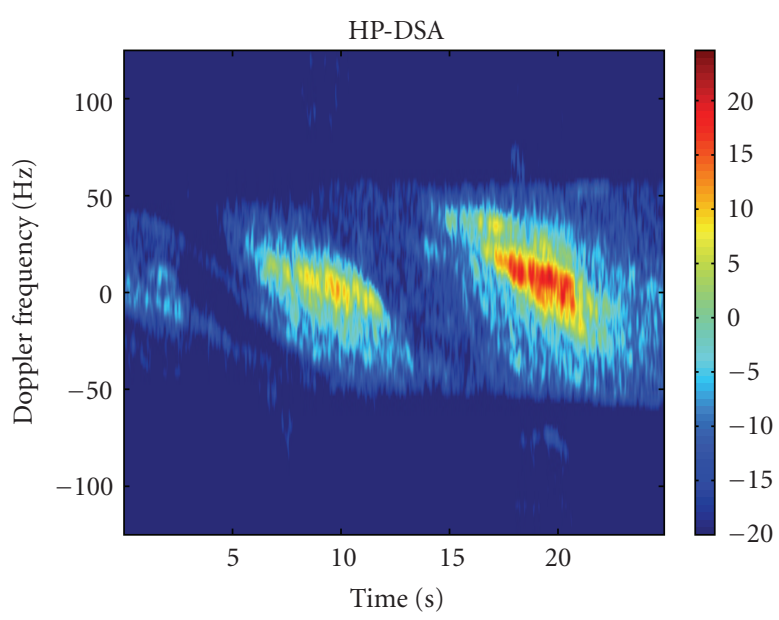

(b)

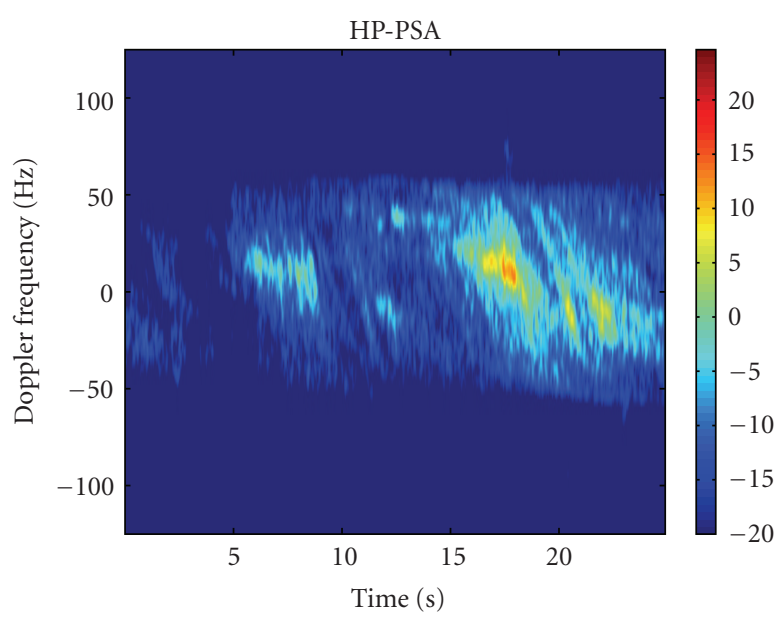

(d)

Figure 7: Doppler power (dB) spectra measured at the VP-DSA, HP-DSA, VP-PSA, and HP-PSA on Kent Street.

reflection from the vertical walls. In contrast, the secondary signal component arriving from the alley is depolarized with nearly equal signal energy on both polarizations of the PSA. The reduced signal energy emitted at $90^{\circ}$ into the street from the alley $(7-8 s)$ is evident again at the PSA; the vehicle shadows most of this signal component from the DSA. On the DSA, there is increasing signal energy from the front as the vehicle moves along Slater Street, which is entering the urban canyon from Bank Street and being reflected from the buildings on either side. Observe also the weaker multipath components, particularly notable on the VP-DSA in the last two seconds of the measurement; these are signals reflected from objects, such as parked cars and buildings, along the side of the street. Although similar objects are present on the other side of the street as well, those on the passenger side are not illuminated as strongly by the energy entering from Bank Street.

The Doppler spectra obtained at the TA on Kent Street are shown in Figure 6 . On this measurement route, the vehicle started near the intersection with Nepean Street, passing through intersections with Gloucester Street (8-12 s) and Laurier Avenue (18-22s). Signal energy reaches Kent
Street predominantly via these intersections. For the first $3 \mathrm{~s}$ of the measurement the area to the west of the vehicle is open and the TA receives signal energy weakly from all directions. However, as the vehicle enters into the shadow of a church located in the centre of the block between Nepean Street and Gloucester Street the power received from the side drops off (3-5s). Nearing the intersection Kent Street and Gloucester Street intersection the power received from broadside to the vehicle increases until, in the centre of the intersection, it dominates the power received. As the vehicle exits the intersection and enters the shadow of the buildings along Kent Street the power from the side decreases once again while the power at the front and rear persists. This pattern is repeated at the Laurier Avenue intersection, however, the power arriving from the Laurier Avenue street canyon is much higher than that received from the Gloucester Street intersection.

The Doppler spectra of the channels measured at the SAs on Kent Street are shown in Figure 7. In contrast to the TA, which received multipath energy from all azimuthal directions, less power is received from the front and rear of the vehicle at the SAs. As the signal energy arrives from 
the intersecting streets, the power in the signal components travelling along the street is much weaker on Kent Street than on Slater Street. At the TA, the signal components arriving from the front and rear for most of the measurement likely result from a combination of diffraction around buildings on the intersection corners and reflection from traffic and buildings along the street. At the SA, however, the lower height of the arrays, the directionality of the SA elements, and shadowing by the vehicle contribute to a lower received power from the front and rear.

As at the TA, the power at the DSA increases as the mobile approaches the intersection with Gloucester Street (6-9s) where strong signal components radiating out of the street are received nearly perpendicular to the array. Shadowing from the vehicle reduces the power received at the PSA, however, the power increases slightly as a result of signal energy reflecting from the building on the north-east side of the intersection, observed as a positive Doppler offset. In the intersection (9-11s), the power received at the PSA is significantly reduced. This is attributed to a reduction in reflected energy due to the open street at the passenger side of the vehicle. A similar effect is observed at the Laurier Avenue intersection (18-22 s), but the received signal power is higher due to the more direct signal path along Laurier Avenue from the transmitter. On both the DSA and PSA, the signal power received on the HP follows a similar pattern to that on the VP, although the average signal power is approximately $5 \mathrm{~dB}$ lower.

The Doppler spectra discussed above reveal that, depending on the location and orientation of the vehicle relative to the transmitter, significant differences may exist in the multipath structure observed at each array position. The potential for MIMO gains to be achieved depends on both the spatial diversity and the received signal power; these are examined in the following sections.

\section{Received Power}

As was seen in Section 3, when the antenna elements are positioned on different sides of the vehicle, shadowing from the vehicle combined with the directionality of the array elements reduces the power received in some multipath components while amplifying the power in others. The received power at each of the side arrays is dependent on the location of the vehicle and the direction it is travelling. This dependency is investigated in this section.

The time series of received power estimates at each array was calculated by applying a length- $N$ sliding window to the measured channel matrices such that

$$
P^{(i, j)}=\frac{1}{N_{t} N_{r} N} \sum_{n=1}^{N}\left\|\mathbf{H}^{(i, j)}[n]\right\|_{F}^{2},
$$

where, as before, $i=\{v, h\}$ and $j=\{T, D, P\}$. The length of the window, $N$, was selected to be equivalent to $13 \lambda$ in accordance with the wide-sense stationarity measurements discussed in Section 2.1.

4.1. Top Array versus Side Arrays. Figure 8 shows the received channel power measured on each array along Slater Street.
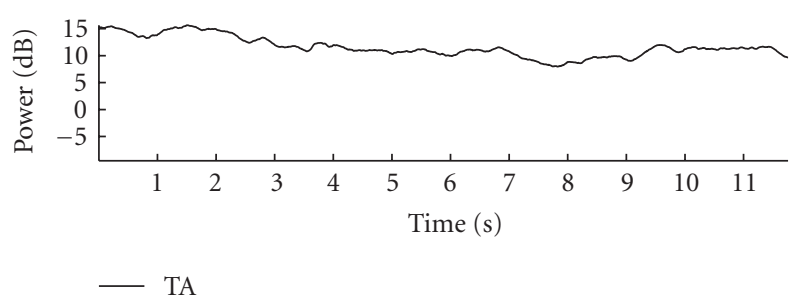

(a)

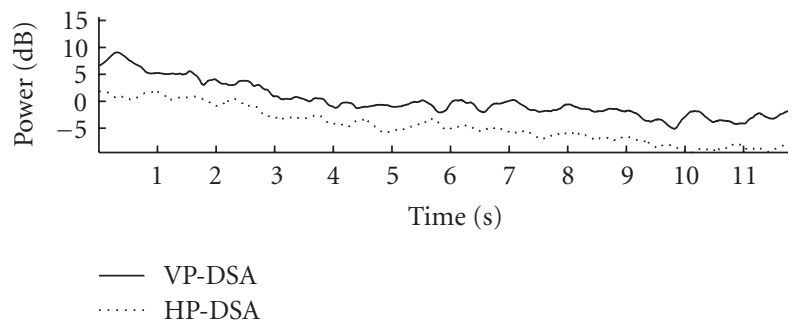

(b)

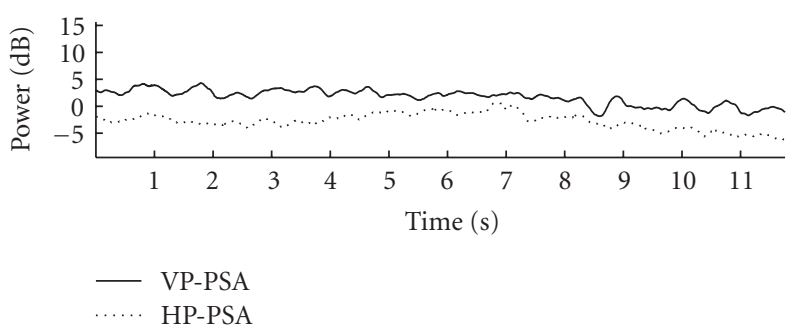

(c)

FigURE 8: Received power measured at each array along Slater Street.

The power received at the TA was, on average, $10 \mathrm{~dB}$ higher than at either VP side array and $15 \mathrm{~dB}$ higher than the HP side arrays.

On this measurement route, as the receiver moves away from the transmitter, the received power decays at a different rate at each array position. The power received on the DSA decreases quite rapidly over the first three seconds, as the distance between the vehicle and the intersection with Kent Street increases. This, plus the directional information in Figure 5, suggests that most of the power received at the two polarizations of the DSA in this interval results from reflection of signal energy arriving along Kent Street from the buildings on the north side of Slater Street. In contrast, the power on the VP-PSA remains fairly constant over the first $8 \mathrm{~s}$; recall that there is a secondary source of power through an alley on the south side of Slater Street. On the HP-PSA, there is very little signal power received from the rear, as seen in Figure 5, and the signal received via the alley (7-8 s) results in a maximum power as the vehicle reaches the alley opening.

A plot of the powers measured on Kent Street is shown in Figure 9. As discussed in Section 3, on this measurement route, signal energy is radiating from the direction of the transmitter along the intersecting street canyons. Within the intersections (the intervals between 8-12 s and 18-22 s), the total received power is dominated by energy propagating along the street canyons. In these regions, the power received 


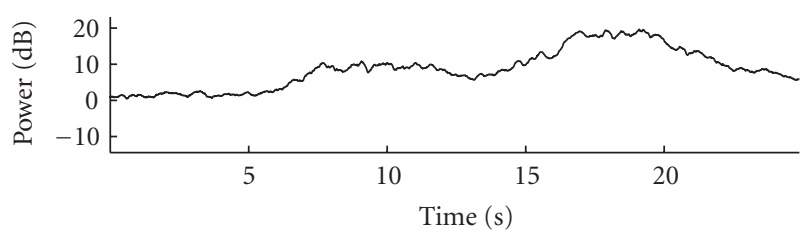

- TA

(a)

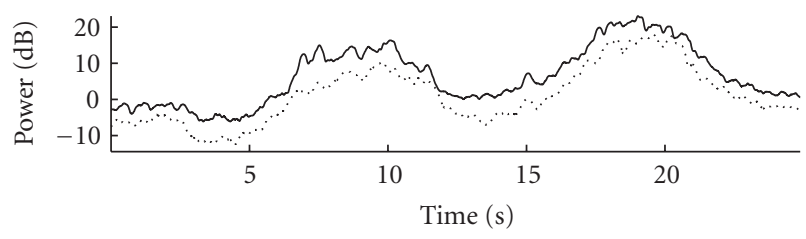

- VP-DSA

… HP-DSA

(b)

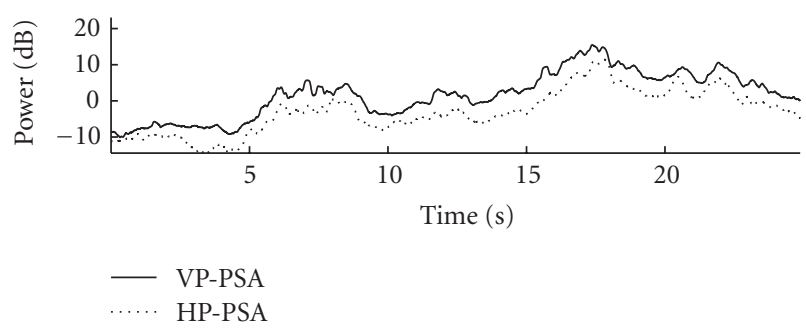

(c)

FIgURE 9: Received power measured at each array along Kent Street.

at the DSA is higher than that measured at the TA due to the directional gain pattern of the patch antennas.

The PSA is shadowed by the vehicle from the signal energy arriving along the intersecting streets. However, increases in power are observed on the VP-PSA and the HP-PSA as the vehicle enters and leaves the intersections. As observed in Figure 7, reflections from the buildings on the east side of the intersection, particularly the northeast corner, direct power to the PSA, resulting in a "U-" shaped power profile with a minimum in the centre of the intersections, which is not observed at the TA.

Between intersections, the incoming signal energy arrives at the receive arrays as scattered and reflected energy from the surrounding buildings on both sides of the street. The TA receives about $6 \mathrm{~dB}$ higher power than either the DSA or PSA in these regions. This is because it has an azimuthal observation of $360^{\circ}$, while the SAs are shadowed from signals arriving on the opposite side of the vehicle. Further, as noted in Section 2, the $10 \mathrm{~dB}$ beamwidth of the patch antennas is limited to $134^{\circ}$, which means that signal components arriving from near the axial directions of the array, that is, the front and rear of the vehicle, are highly attenuated.

4.2. Driver Side versus Passenger Side. To quantify the difference in power received at the two side array positions, the array power imbalance (API) metric is defined for a given polarization as the ratio of the powers measured at the driver and passenger side of the vehicle, that is,

$$
\Lambda^{(i)}=\frac{\sum_{n=1}^{N}\left\|\mathbf{H}^{(i, D)}[n]\right\|_{F}^{2}}{\sum_{n=1}^{N}\left\|\mathbf{H}^{(i, P)}[n]\right\|_{F}^{2}}=\frac{P^{(i, D)}}{P^{(i, P)}} .
$$

Note that the powers are averaged over all the antenna elements in each array, and a length- $N$ sliding window equivalent to $13 \lambda$ is applied as before.

The API measured on Slater Street and Kent Street is shown in Figure 10. Slater Street exhibited the lowest APIs of all the routes measured with an average value of $-2 \mathrm{~dB}$. As noted in Figure 8, the power on the DSA decreases over the first $3 \mathrm{~s}$; this is reflected in the API shown in Figure 10(a). Beyond $3 \mathrm{~s}$, the average power on the PSA exceeds that on the DSA, for both polarizations, due mainly to the secondary power source at 7-8 s. In contrast, the API measured on Kent Street, Figure 10(b), shows significant variation in the API between the intersections and the intrablock regions. In the intersections, where the vehicle shadows the PSA from the directional power arriving along the intersecting streets, the API exceeds $16 \mathrm{~dB}$. Note the API peaks are narrower than the peaks in DSA power observed in Figure 9 due to the "U-" shape of the power profile at the PSA, as described above. Outside the intersections, the power received at both sides of the vehicle results from signal components reflected from buildings along the street. The power in these regions is more evenly distributed at each SA position, and the API is closer to unity.

4.3. Vertical versus Horizontal Polarization. The power imbalance between received signal polarizations is parameterized as cross polarization discrimination (XPD). In this case, the signal was transmitted only on the vertical polarization; hence, the XPD quantifies the degree to which the signal became depolarized as it was reflected, scattered and diffracted by objects in the physical environment.

The XPD with respect to the vertical polarization was computed as

$$
\bar{\chi}^{(j)}=\frac{\sum_{n=1}^{N}\left\|\mathbf{H}^{(v, j)}[n]\right\|_{F}^{2}}{\sum_{n=1}^{N}\left\|\mathbf{H}^{(h, j)}[n]\right\|_{F}^{2}}=\frac{P^{(v, j)}}{P^{(h, j)}},
$$

where, again, the length of the average, $N$, was equivalent to approximately $13 \lambda$ for all the arrays.

The time series of the XPD measured at the driver side and passenger side on Slater Street and Kent Street are shown in Figure 11. For the whole of these measurements, the average received power on the VP exceeded that on the HP; this is expected due to the polarization of the transmitted signal and because of the vertical structure of the urban environment, where reflection and diffraction occur predominantly from and around vertical surfaces and corners, limiting depolarization. At the DSA on Slater Street, after an initial peak, possibly due to a strong component diffracted at the corner with Kent Street and hence vertically polarized, the XPD remains fairly consistent. On the PSA; however, the XPD decreases as the vehicle approaches the alley on the south side of Slater Street, and then decreases 


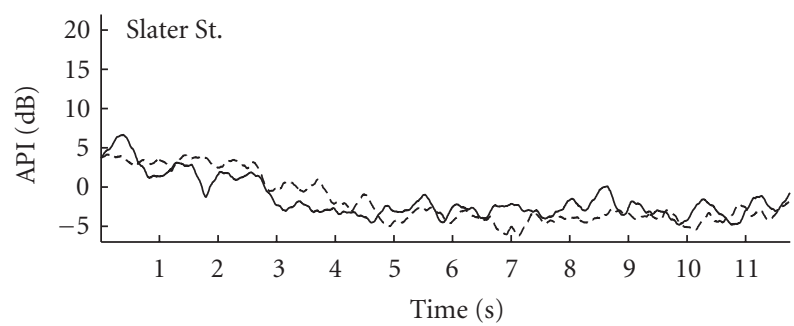

- VP

(a)

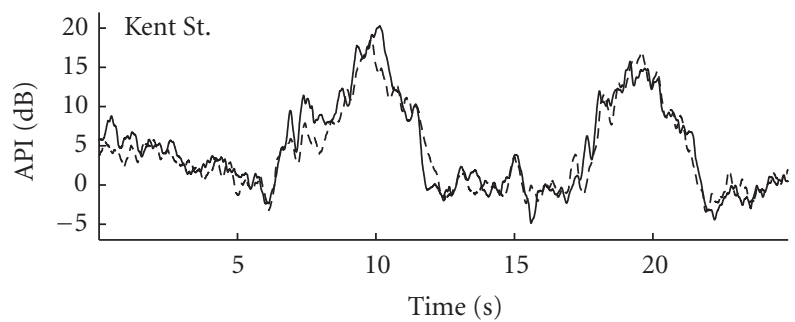

- VP

(b)

Figure 10: API measured on Slater Street (a) and Kent Street (b).

after; this suggests the signal emitted from the alley is more depolarized than that propagating along Slater Street from the intersection at Kent Street.

On Kent Street, the XPD at the DSA increases markedly as the vehicle enters and exits the intersections, but is lower inside the intersection. This suggests that a signal component is reflected off the surface of the intersecting street within the intersection, thus a strong HP component is received. At the edges of the intersection, some of the power arrives via a mechanism favouring vertical copolarization, such as diffraction around the building corners or reflection from their walls. This effect is reduced at the PSA as the component along the intersecting street is shadowed by the vehicle.

In general, similar average depolarization characteristics were measured at each side of the vehicle. On all of the measurement routes, $90 \%$ of the time $\bar{\chi}^{(v, j)}$ measured on one side of the vehicle was within $0-6 \mathrm{~dB}$ of that measured at the other side.

\section{Diversity}

As observed in Section 3, the received multipath components depend on the position of the array on the vehicle and the orientation of the vehicle on the street. This can have a significant impact on the diversity characteristics, which are investigated in this section through the diversity metric [43]

$$
\Phi^{(i, j)}=\frac{\operatorname{tr}\left\{\mathbf{R}^{(i, j)}\right\}^{2}}{\left\|\mathbf{R}^{(i, j)}\right\|_{F}^{2}}=\frac{\left[\sum_{\ell=1}^{N_{r}} \sigma_{\ell}\right]^{2}}{\sum_{\ell=1}^{N_{r}} \sigma_{\ell}^{2}},
$$

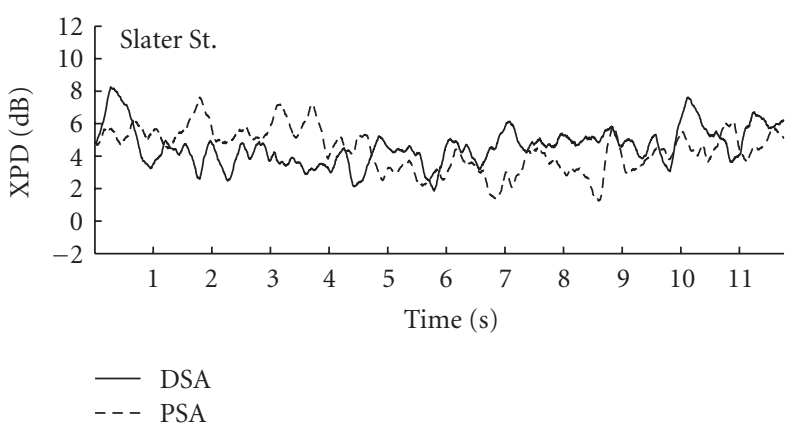

(a)

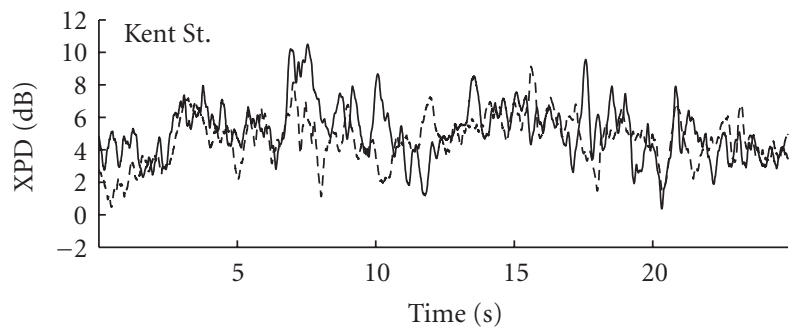

$\begin{array}{ll}- & \text { DSA } \\ -- & \text { PSA }\end{array}$

(b)

Figure 11: Average XPD measured on Slater Street (a) and Kent Street (b).

where $\operatorname{tr}\{\cdot\}$ is the trace of the matrix argument and $\sigma_{\ell}$, $\ell=1, \ldots, N_{r}$, is the $\ell$ th eigenvalue of the estimated autocorrelation matrix

$$
\mathbf{R}^{(i, j)}=\frac{1}{N} \sum_{n=1}^{N} \mathbf{H}^{(i, j)}[n] \mathbf{H}^{(i, j)^{H}}[n] .
$$

As noted previously, the value of $N$ corresponds to approximately $2 \mathrm{~m}$ or $13 \lambda$. In highly correlated channels, the eigenspectrum contains a small number of significant eigenvalues and $\Phi \rightarrow 1$. Alternatively, when the channel is rich in angularly distributed multipath components such that the propagation paths between each transmit-receive element pair are independent, the eigenvalues will be more uniformly distributed and $\Phi \rightarrow \min \left(N_{r}, N_{t}\right)$.

The diversity measured at each array on Slater Street is shown in Figure 12. At the TA, the diversity is high initially and then drops at around $2 \mathrm{~s}$, where the dominant signal arrives from near the rear of the vehicle, see Figure 4. As the signal energy from the secondary source, the alley between Laurier Avenue and Slater Street, increases and moves from the front to the side of the vehicle, the diversity again exceeds three. There is a slight decrease as the vehicle passes the alley, corresponding to the loss of that signal as discussed in Section 3. After the vehicle has passed the alley, almost all the signal power is being received from the rear, resulting in a loss of spatial diversity.

At the VP-DSA, the diversity is low over the first $3 \mathrm{~s}$, when there is a dominant signal component arriving from the rear of the vehicle. Beyond this, the diversity remains relatively 


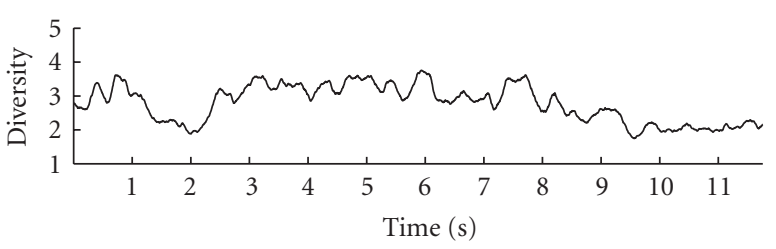

(a)

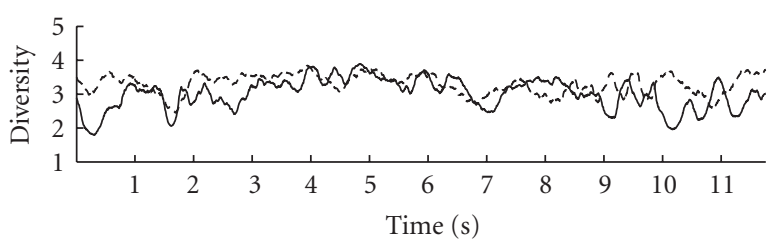

VP-DSA

- - HP-DSA

(b)

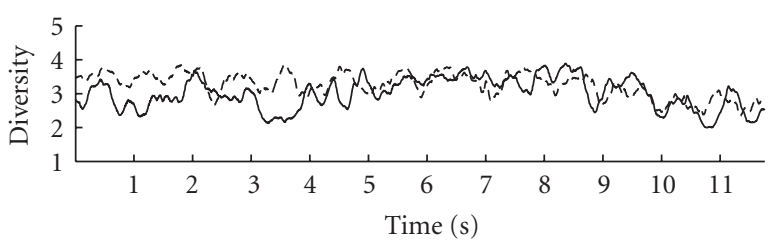

VP-PSA

- - HP-PSA

(c)

FIGURE 12: Diversity measure of the channels at each array on Slater Street.

constant until around $9 \mathrm{~s}$ : although the total received power is low (see Figure 5(a)), the power is received over a wide angular range. Over the last $3 \mathrm{~s}$, the contributions from the front are reduced, and the diversity drops. The diversity on the HP-DSA is fairly constant over the whole measurement because the angular range of the weak multipath components is quite large, and there is no dominating component, resulting in eigenvalues that are more uniform.

On the VP-PSA, the diversity is highest in the approach to the alley opening, when there are several multipath components arriving with large angular spread (see Figure $5(\mathrm{c})$ ). As on the TA, past the opening, the diversity drops because most of the signal energy is coming from a single direction, resulting in an autocorrelation matrix $\mathbf{R}$ with fewer dominant eigenvalues. The HP-PSA follows a similar trend, except the diversity is higher over the first $4 \mathrm{~s}$ because the signal arriving from near the rear of the vehicle is not as strong compared to the other signal components, and therefore, the eigenvalues are more uniform.

The diversity, computed using (9), for Kent Sreet is shown in Figure 13. At both the TA and the DSA, the diversity is highest outside the intersections, where the signal arrives from a wide range of angles (see Figures 6 and 7). In the intersections, most of the signal power arrives from along the intersecting street, over a fairly narrow angle, resulting in a dominant eigenvalue and low diversity. Note that this

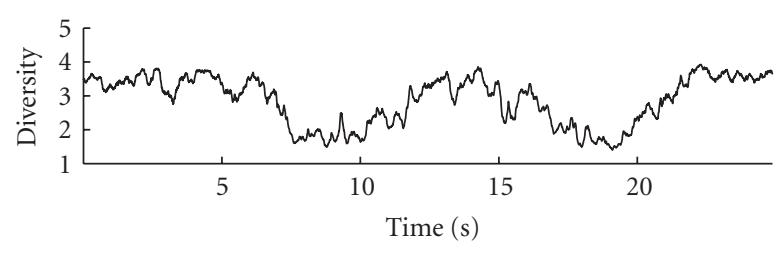

- TA

(a)

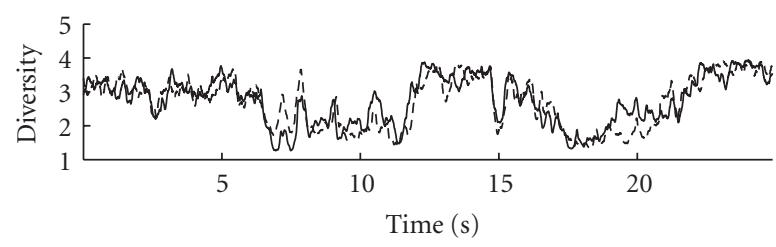

VP-DSA

(b)

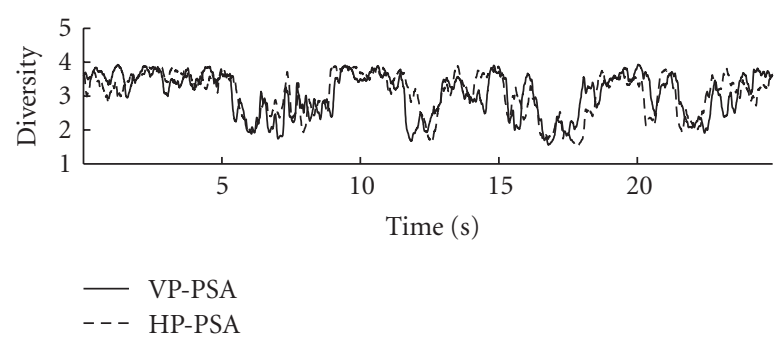

(c)

FIGURE 13: Diversity measure of the channels at each array on Kent Street.

is opposite to the received power characteristics observed in Section 4.1. At the PSA, the diversity is lowest at the entry and exit to the intersections, where the power is again received from a narrow range of angles, in this case, the buildings on the north-east corner of the intersection. In the centre of the intersection and between intersections, the diversity is high, reflecting the fact that the power, although weak, arrives over a range of angles. The HP arrays have similar diversity characteristics to their VP counterparts.

\section{Capacity}

Although capacity is not a complete indicator of how a system will perform in a given environment, it does provide an upper bound on the maximum achievable throughput. The ergodic capacity of a MIMO system without channel state information at the transmitter is defined as [44],

$$
C=E\left\{\log _{2}\left(\operatorname{det}\left[\mathbf{I}_{N_{r}}+\frac{\gamma}{N_{t}} \mathbf{H H}^{H}\right]\right)\right\} \mathrm{b} / \mathrm{s} / \mathrm{Hz},
$$

where $\operatorname{det}[\cdot]$ is the determinate, $\gamma$ is the SNR, $\mathbf{I}_{N_{r}}$ is the $N_{r} \times$ $N_{r}$ identity matrix, and the expectation $E\{\cdot\}$ here is taken over a distance of $13 \lambda$.

The capacity estimated at each array on Slater street and Kent Street is shown in Figures 14 and 15, respectively, and 


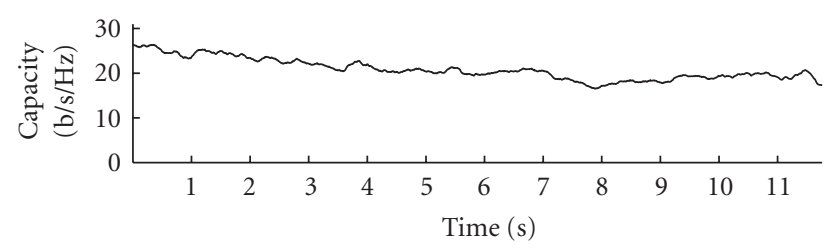

- TA

(a)
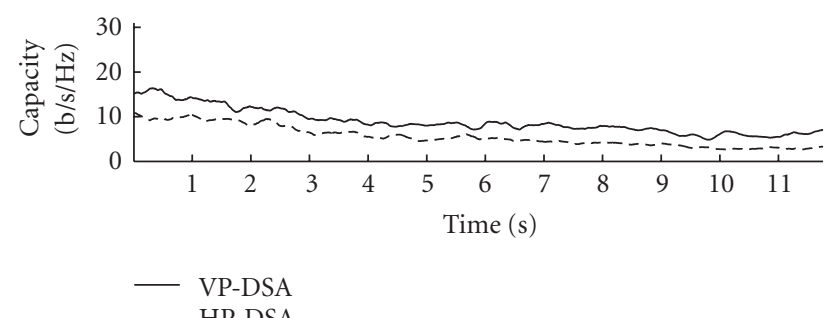

- - HP-DSA

(b)

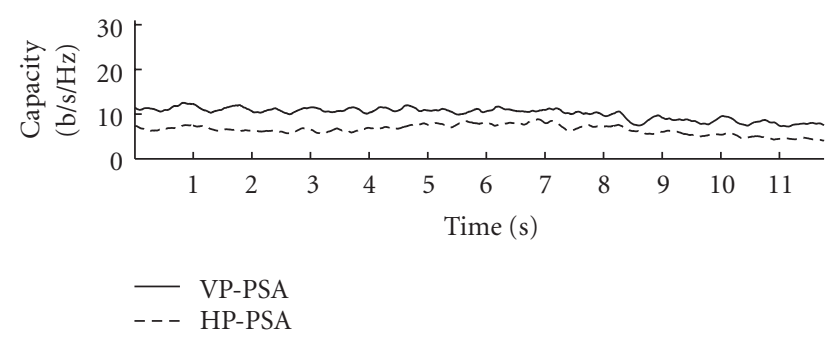

(c)

FIgURe 14: Capacity estimated at each array position on Slater Street.

estimated based on a total average SNR of $20 \mathrm{~dB}$ at the TA. The trends follow the power measurements, shown in Figures 8 and 9, quite closely. The increased diversity has only a small impact, mostly at low received power, that can be observed in the difference between the polarizations on the DSA in the last few seconds of the measurement on Slater Street and on both sides of the vehicle in the first $5 \mathrm{~s}$ on Kent Street. The capacity on the TA is much larger than that on the SAs on both measurements, due to the higher power received.

Metrics that have been proposed for MIMO channel model validation include spatial structure in the form of angular or Doppler power spectra, diversity, and capacity [45]. From Figures 14 and 15, it is clear that the capacity is dominated by received power and is not very sensitive to other channel characteristics. Antenna array elements in different positions on the vehicle receive different average powers in given locations; hence, average received power per element is a more suitable metric for validating MIMO channel models for these scenarios.

\section{Conclusions}

In vehicular-based MIMO systems, the position of the array on the vehicle is critical to the system performance. Antenna elements placed on the vehicle sides receive only a subset of the multipath components received by roof-mounted arrays

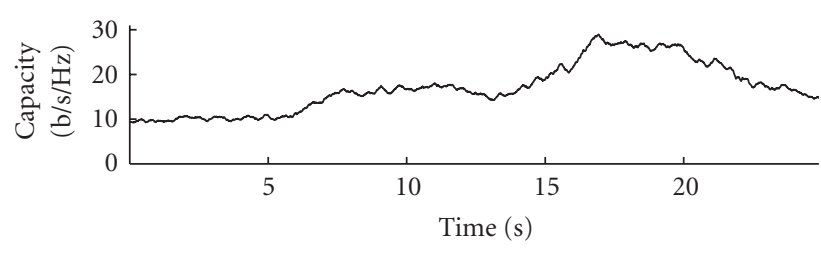

- TA

(a)

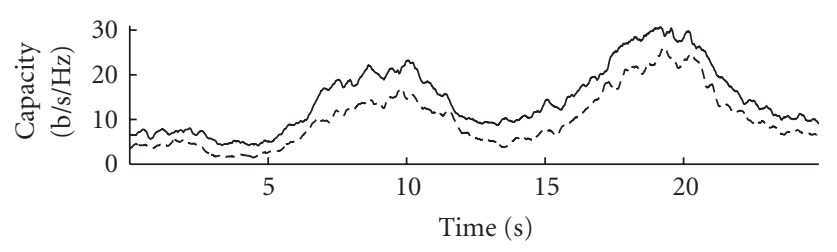

- VP-DSA

-- - HP-DSA

(b)

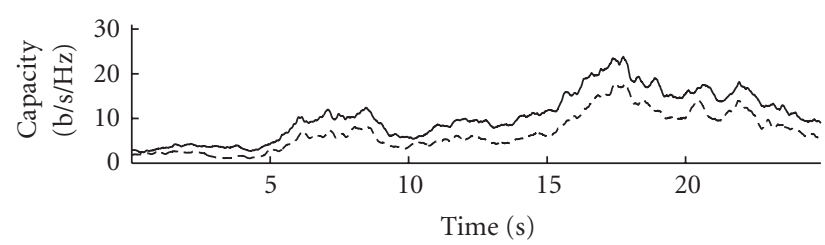

$\begin{array}{ll}- & \text { VP-PSA } \\ --- & \text { HP-PSA }\end{array}$

(c)

FIGURE 15: Capacity estimated at each array position on Kent Street.

due to their reduced beamwidth and shadowing by the vehicle. As a result, the power received at a side-mounted array will generally be $6-10 \mathrm{~dB}$ less than at a roof-mounted array except for situations where the side arrays are pointed toward the transmitter. In these scenarios, the difference in the power received at each side array was measured to be as high as $20 \mathrm{~dB}$. However, when a strong, directional multipath component reduces the diversity at one array, the diversity at the other arrays may be higher. In terms of theoretical channel capacity, the differences in diversity will generally be eclipsed by the larger received power.

The wide variation in received power indicates that a MIMO system with side-mounted receiver arrays will likely require either higher receiver sensitivity or higher transmit power, and a larger dynamic range, than a system with antennas mounted on the top of the vehicle.

In general, from a capacity perspective, the best performance results from array elements mounted on the top of the vehicle, that is, where the exposure of the array elements to incoming multipath components is maximized. However, if such an arrangement is restricted, the array elements should be distributed around the vehicle to ensure that sufficient signal power is received on, at minimum, a subset of the array elements. While this may result in a power imbalance among the array elements, it will limit the receiver's susceptibility 
to shadowing by the vehicle and help increase multipath diversity.

Most analytical MIMO channel models are parameterized based on the assumption that the effective channel characteristics observed at each array element are similar. However, the Doppler spectra in Section 3 show that significant differences in observed propagation characteristics may exist between the sides and top of a vehicle which are not accounted for by typical models.

An additional challenge arises if the antenna elements are positioned around the vehicle; in this case, modified channel models must be derived that account for differences in the observed propagation characteristics at each array position at any given time. In particular, analytical channel models would have to take into account the differences in received power at each antenna element.

For path-based MIMO channel models, the directionality of the multipath components plays a critical role in both received power and diversity, and, hence, in system capacity. The observations described in the preceding sections indicate that the characteristics of the channel are highly dependent on location; for example, features at urban intersections result in markedly different received signal structures than those in urban canyons. The challenge for a path-based model is to place clusters in realistic locations to replicate these effects.

As many measurements have been made in the last decade using roof-mounted antenna arrays, it is interesting to consider whether those measurements can be used to derive suitable models of antenna elements positioned elsewhere on the vehicle, for example, using detailed radiation patterns of the vehicle and antennas combined. The observations from this work show that the vehicle itself shadows, or obstructs, signal components which might otherwise be reflected or scattered on the far side from the transmitter before being received by antenna elements on that side. It is also a reflective object which may increase signal components on the near side. To derive an appropriate model from measurements made using typical roof-mounted arrays alone would require identifying which portion of the received energy would have been shadowed and removing it, and where signal energy would be reflected and adding it. This is not considered to be a realistic approach, particularly using measurements from the type of roof-mounted array used in this work, which does not provide an omnidirectional pattern. Further, the roof-mounted array is approximately twice as high above street level as the side-mounted array, which may introduce further inaccuracies.

Further experimental work is required to investigate the impact of antenna position for different vehicles and to use for the derivation and the validation of channel models. Significant differences in the propagation characteristics have been observed when array elements are mounted at different vehicular positions, and these should be taken into account when designing MIMO systems for vehicular applications. This requires appropriate models that take into account the instantaneous differences observed at each array position, so the available diversity can be fully exploited.

\section{Acknowledgment}

The authors thank the CRC Advanced Antenna Technology group for designing the patch antennas used in this study.

\section{References}

[1] G. J. Foschini, "Layered space-time architecture for wireless communication in a fading environment when using multielement antennas," Bell Labs Technical Journal, vol. 1, no. 2, pp. 41-59, 1996.

[2] S. M. Alamouti, "A simple transmit diversity technique for wireless communications," IEEE Journal on Selected Areas in Communications, vol. 16, no. 8, pp. 1451-1458, 1998.

[3] A. Paulraj, R. Nabar, and D. Gore, Introduction to Space-Time Wireless Communications, Cambridge University Press, New York, NY, USA, 2003.

[4] G. D. Durgin and T. S. Rappaport, "Effects of multipath angular spread on the spatial cross-correlation of received voltage envelopes," in Proceedings of the 49th IEEE Vehicular Technology (VTC'99), pp. 996-1000, May 1999.

[5] M. Pätzold and B. O. Hogstad, "A wideband MIMO channel model derived from the geometric elliptical scattering model," Wireless Communications and Mobile Computing, vol. 8, no. 5, pp. 597-605, 2008.

[6] A. F. Molisch, A. Kuchar, J. Laurila, K. Hugl, and R. Schmalenberger, "Geometry-based directional model for mobile radio channels_-principles and implementation," European Transactions on Telecommunications, vol. 14, no. 4, pp. 351-359, 2003.

[7] S. Haykin and K. J. R. Lui, Eds., Handbook on Array Processing and Sensor Networks, Wiley-IEEE Press, New York, NY, USA, 2009.

[8] W. Roh and A. Paulraj, "MIMO channel capacity for the distributed antenna systems," in Proceedings of the 56th IEEE Vehicular Technology (VTC FALL '02), vol. 2, pp. 706-709, September 2002.

[9] C. C. Martin, J. H. Winters, and N. R. Sollenberger, "MIMO radio channel measurements: performance comparison of antenna configurations," in Proceedings of the 54th IEEE Vehicular Technology (VTC FALL '01), vol. 2, pp. 1225-1229, October 2001.

[10] M. Batariere, T. Blankenship, J. Kepler et al., "Wideband MIMO mobile impulse response measurements at $3.7 \mathrm{GHz}$," in Proceedings of the 55th IEEE Vehicular Technology (VTC '02), vol. 1, pp. 26-30, 2002.

[11] D. Chizhik, J. Ling, P. Wolniansky, R. Valenzuela, N. Costa, and K. Huber, "Multiple-input-multiple-output measurements and modeling in Manhattan," IEEE Journal on Selected Areas in Communications, vol. 21, no. 3, pp. 321-331, 2003.

[12] C. C. Squires, T. J. Willink, and B. Gagnon, "A flexible platform for MIMO channel characterisation and system evaluation," in Proceedings of the 15th International Conference on Wireless Communication (WIRELESS '03), vol. 2, pp. 441-450, July 2003.

[13] K. Nishimori, Y. Makise, M. Ida, R. Kudo, and K. Tsunekawa, "Channel capacity measurement of $8 \times 2$ MIMO transmission by antenna configurations in an actual cellular environment," in Proceedings of the IEEE Transactions on Antennas and Propagation, vol. 54, pp. 3285-3291, November 2006.

[14] J. Ling, D. Chizhik, D. Samardzija, and R. A. Valenzuela, "Peerto-peer MIMO radio channel measurements in a rural area," 
IEEE Transactions on Wireless Communications, vol. 6, no. 9, pp. 3229-3237, 2007.

[15] T. Murakami, N. Honma, K. Nishimori, R. Kudo, Y. Takatori, and M. Mizoguchi, "Multi site MIMO channel analysis at $4.85 \mathrm{GHz}$ in outdoor environment," in Proceedings of the 20th IEEE International Symposium on Personal, Indoor and Mobile Radio Communications (PIMRC '09), pp. 3005-3009, September 2009.

[16] W. Kim, H. Lee, J. J. Park, M.-D. Kim, and H. K. Chung, "Modeling of eigenvalues for MIMO channel capacity based on outdoor measurements," in Proceedings of the 69th IEEE Vehicular Technology(VTC '09), April 2009.

[17] T. Kan, R. Funada, J. Wang, H. Harada, and J. I. Takada, "MIMO channel capacity of a measured radio channel for outdoor macro cellular systems at $3 \mathrm{GHz}$-band," in Proceedings of the 70th IEEE Vehicular Technology (VTC FALL '09), September 2009.

[18] K. Sulonen, P. Suvikunnas, L. Vuokko, J. Kivinen, and P. Vainikainen, "Comparison of MIMO antenna configurations in picocell and microcell environments," IEEE Journal on Selected Areas in Communications, vol. 21, no. 5, pp. 703-712, 2003.

[19] J. W. Wallace, M. A. Jensen, A. Gummalla, and H. B. Lee, "Experimental characterization of the outdoor MIMO wireless channel temporal variation," IEEE Transactions on Vehicular Technology, vol. 56, no. 3, pp. 1041-1049, 2007.

[20] E. Ohlmer, J. Hofrichter, S. Bittner et al., "Urban outdoor MIMO experiments with realistic handset and base station antennas," in Proceedings of the 71st IEEE Vehicular Technology (VTC'10), pp. 1-5, May 2010.

[21] D. Baum, D. Gore, R. Nabar et al., "Measurement and characterization of broadband MIMO fixed wireless channels at $2.5 \mathrm{GHz}$," in Proceedings of the 11th IEEE International Symposium On Personal, Indoor And Mobile Radio Communication (PIMRC'00), pp. 203-206, September 2000.

[22] M. Gans, N. Amitay, Y. Yeh et al., "Outdoor BLAST measurement system at $2.44 \mathrm{GHz}$ : calibration and initial results," IEEE Journal on Selected Areas in Communications, vol. 20, no. 3, pp. 570-583, 2002.

[23] V. Erceg, P. Soma, D. S. Baum, and S. Catreux, "Multiple-input multiple-output fixed wireless radio channel measurements and modeling using dual-polarized antennas at $2.5 \mathrm{GHz}$," IEEE Transactions on Wireless Communications, vol. 3, no. 6, pp. 2288-2298, 2004.

[24] Y. Yang, G. Xu, and H. Ling, "An experimental investigation of wideband MIMO channel characteristics based on outdoor non-LOS measurements at $1.8 \mathrm{GHz}$," in Proceedings of the IEEE Transactions on Antennas and Propagation, vol. 54, pp. 32743284, November 2006.

[25] R. Valenzuela, L. Ahumada, and R. Feick, "Multi-antenna techniques in fixed wireless links," in Proceedings of the 69th IEEE Vehicular Technology (VTC '09), April 2009.

[26] R. Thomä, D. Hampicke, M. Landmann, G. Sommerkorn, and A. Richter, "MIMO measurement for double-directional 26 channel modelling," in Proceedings of the IEEE Seminar on Communications Systems from Concept to Implementations, December 2001.

[27] J. Liberti, J. Koshy, T. Hoerning et al., "Experimental results using a MIMO test bed for wideband, high spectral efficiency tactical communications," in Proceedings of the IEEE Military Communications (MILCOM '05), vol. 3, pp. 1340-1345, October 2005.
[28] A. Paier, J. Karedal, N. Czink et al., "First results from car-tocar and car-to-infrastructure radio channel measurements at 5.2 GHZ," in Proceedings of the 18th IEEE International Symposium on Personal, Indoor and Mobile Radio Communications (PIMRC '07), September 2007.

[29] J. Karedal, F. Tufvesson, N. Czink et al., "A geometry-based stochastic MIMO model for vehicle-to-vehicle communications," IEEE Transactions on Wireless Communications, vol. 8, no. 7, pp. 3646-3657, 2009.

[30] A. Alonso, A. Paier, T. Zemen, N. Czink, and F. Tufvesson, "Capacity evaluation of measured vehicle-to-vehicle radio channels at $5.2 \mathrm{GHz}$," in Proceedings of the IEEE International Conference on Communications (ICC '10), May 2010.

[31] V. Erceg, H. Sampath, and S. Catreux-Erceg, "Dualpolarization versus single-polarization MIMO channel measurement results and modeling," IEEE Transactions on Wireless Communications, vol. 5, no. 1, pp. 28-33, 2006.

[32] A. F. Molisch, Wireless Communications, John Wiley \& Sons, New York, NY, USA, 2005.

[33] R. J. C. Bultitude, "Estimating frequency correlation functions from propagation measurements on fading radio channels: a critical review," IEEE Journal on Selected Areas in Communications, vol. 20, no. 6, pp. 1133-1143, 2002.

[34] R. J. C. Bultitude, T. C. W. Schenk, N. A. A. O. den Kamp, and N. Adnani, "A propagation-measurement-based evaluation of channel characteristics and models pertinent to the expansion of mobile radio systems to frequencies beyond $2 \mathrm{GHz}$," IEEE Transactions on Vehicular Technology, vol. 56, no. 2, pp. 382388, 2007.

[35] D. Ribbenfjard, B. Lindmark, B. Karlsson, and L. Eklund, "Omnidirectional vehicle antenna for measurement of radio coverage at 2 GHz v.2.0," IEEE Antennas and Wireless Propagation Letters, vol. 3, no. 1, pp. 269-272, 2004.

[36] C. C. Squires, The Impact of Polarized MIMO Channels in Vehicular Based Communications, M.S. thesis, University of Ottawa, Ottawa, Canada, 2009.

[37] C. C. Squires and T. J. Willink, "The impact of vehicular antenna placement on polarization diversity," in Proceedings of the 70th IEEE Vehicular Technology (VTC FALL '09), September 2009.

[38] M. Herdin and E. Bonek, "A MIMO correlation matrix based metric for characterizing non-stationarity," in Proceedings of the 1st Mobile and Wireless Communications, June 2004.

[39] J. W. Wallace and M. A. Jensen, "Time-varying MIMO channels: measurement, analysis, and modeling," in Proceedings of the IEEE Transactions on Antennas and Propagation, vol. 54, pp. 3265-3273, November 2006.

[40] R. J. C. Bultitude, T. J. Willink, M. H. A. J. Herben, and G. Brussaard, "Detection of changes in the spectral characteristics of measured mobile radio data for space wave modelling applications," in Proceedings of the Queen's University, Biennial Symposium on Communications, pp. 90-94, Kingston, Canada, May 2000.

[41] T. J. Willink, "Wide-sense stationarity of mobile MIMO radio channels," IEEE Transactions on Vehicular Technology, vol. 57, no. 2, pp. 704-714, 2008.

[42] J. Proakis and D. Manolakis, Digital Signal Processing, Pearson Prentice Hall, 2007.

[43] T. J. Willink, "Characteristics of urban vehicular MIMO channels at different frequencies," in Proceedings of the $3 \mathrm{rd}$ European Conference on Antennas and Propagation (EuCAP '09), pp. 645-649, March 2009. 
[44] I. E. Telatar, "Capacity of multi-antenna Gaussian channels," European Transactions on Telecommunications, vol. 10, no. 6, pp. 585-595, 1999.

[45] H. Özcelik, N. Czink, and E. Bonek, "What makes a good MIMO channel model?" in Proceedings of the 61st Vehicular Technology (VTC '05), vol. 1, pp. 156-160, Stockholm, Sweden, May 2005. 

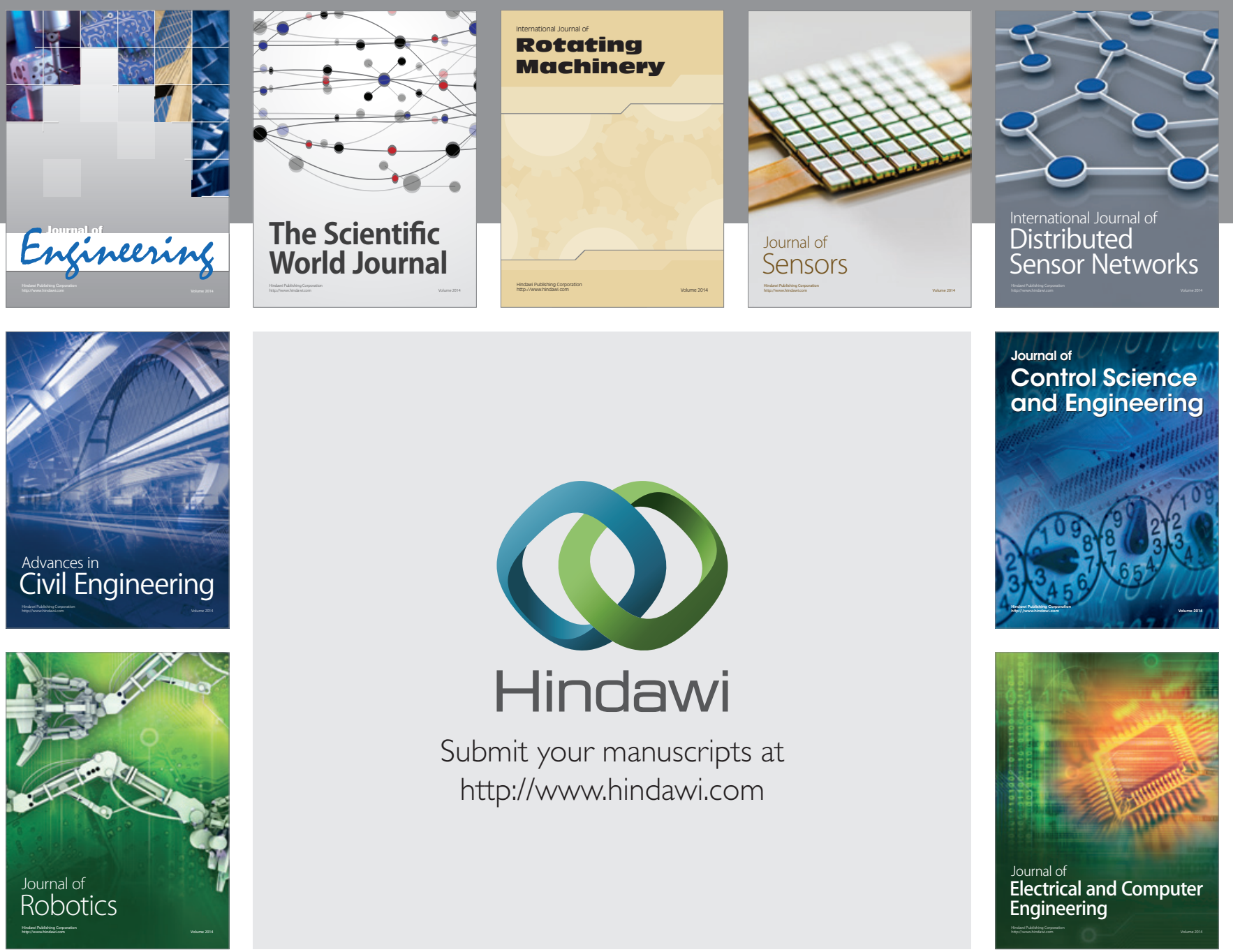

Submit your manuscripts at

http://www.hindawi.com
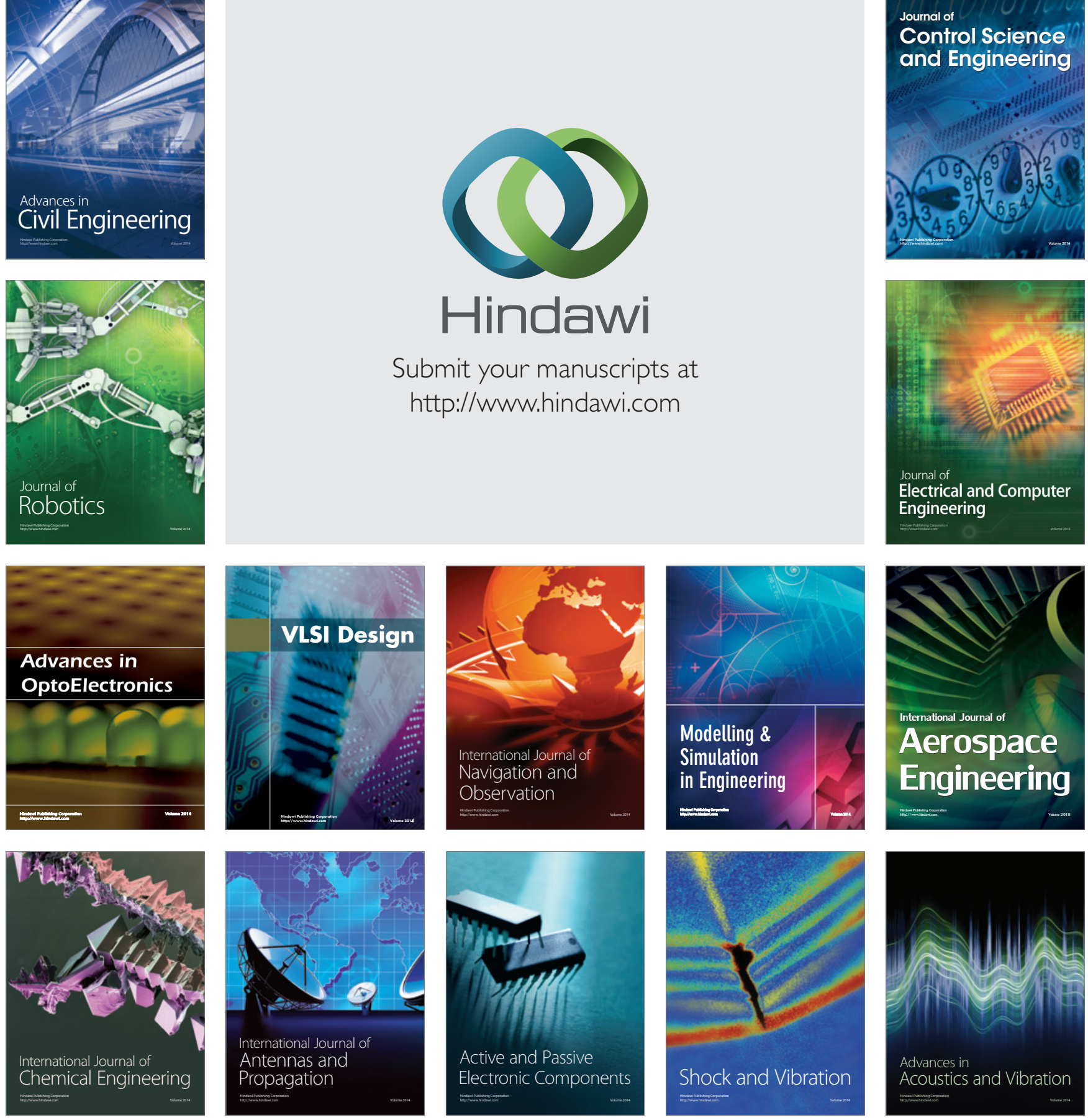